\title{
Bezeichnenderweise Eco
}

Martin Siefkes, Technische Universität Chemnitz Veronika Opletalová, Palacký-Universität Olmütz Emanuele Arielli, Universität IUAV in Venedig

Mit Umberto Eco hat die Semiotik einen der originellsten Zeichentheoretiker und zugleich einen Zeichen setzenden Intellektuellen verloren. Dies ist mehr als eine Metapher: Ecos Analysen zeitgenössischer Kulturphänomene gingen so weit, dass er durch sie die betreffenden Kulturen veränderte. Er nahm als einer der ersten die Popkultur des späten zwanzigsten Jahrhunderts vom Krimi bis zum Comic unter die (semiotische) Lupe und wurde damit schließlich selbst zu einem Teil der Gegenwartskultur. Glaubt man gewissen Rankings, dann war Eco, der verschmitzte Analytiker der Massenkultur, gegen Ende seines Lebens ein weltweiter Popstar der Wissenschaft wie Chomsky oder Dawkins ${ }^{1}$ - eine Volte des publizistischen Schicksals, deren Ironie dem erfahrenen Verlagsmenschen sicher nicht entgangen ist.

Dabei war Ecos intellektueller Stil weniger der des wissenschaftlichen Revoluzzers, der mit neuen Theorien vorprescht und diese dann jahrzehntelang verbissen verteidigt. Eher war er der leidenschaftliche Archivar, dessen Überlegungen auf einem synkretistischen Verständnis von Wissenschaft beruhten. Schaut man in die Inhaltsverzeichnisse seiner berühmten semiotischen Grundlagenwerke La struttura assente (Eco 1968), A Theory of Semiotics (Eco 1976) und Semiotics and the Philosophy of Language (Eco 1984a), dann wirken diese wie nach dem Baukastenprinzip aus bereits etablierten semiotisch-linguistischen Begriffen wie „Zeichen“, „Kommunikation“, „Symbol“, „Metapher“, „Code“ und „Isotopie“ zusammengesetzt. Man ist versucht, von einer Theorie der Semiotik zu sprechen, die wie eine Ausstellung aus allerlei semiotischen Gegenständen kuratiert wurde. Bei der Lektüre wird dann jedoch deutlich, dass man es mit originellen Theoriegebäuden von großer Tragweite zu tun hat, die ihre Herkunft aus den semiotischen Klassikern der ersten Hälfte des 20. Jahrhunderts und aus dem Strukturalismus, der damals kontrovers diskutiert wurde und zu dem Eco ein ambivalentes Verhältnis hatte (er lehnte den „ontologischen Strukturalismus" ab und befürwortete einen methodologischen Struktura- 
lismus, vgl. Eco 1968), nicht verleugnen. Voraussetzung für diese, großen Würfe" war Ecos enormes Wissen, seine Bereitschaft, sich tiefgehend in vorhandene Ansätze einzuarbeiten und das Gefundene mit einer gewissen Frechheit neu anzuordnen und manchmal auch umzudeuten.

In ähnlicher Weise versuchte er den Kulturen des Gestern und des Heute dadurch gerecht zu werden, dass er sie in virtuellen Archiven sammelte. Die Frage, wie man sich im tradierten Wissen orientiert, beschäftigte inn lebenslang und führte zu Sammlungen von Listen, Beschreibungen von Mechanismen der Wissensorganisation und Skizzen von Bibliotheken. Er bezeichnete sich selbst als wandelnde Bibliothek, und seine Privatbibliothek soll 50.000 Bände umfassen. Der internationale Erfolg insbesondere von Ecos Literatur macht es vorstellbar, dass sein Werk unter Berücksichtigung von Neuauflagen und Übersetzungen in nicht allzu langer Zeit ein Bibliothekslabyrinth füllen würde, wie er es für Der Name der Rose gezeichnet hat. Statt seine Schriften aber in ein fiktives Bauwerk einzusortieren, wollen wir uns im Folgenden lieber am Semiotik-Archiv orientieren, das an der Universität der Künste in Berlin angesiedelt ist und auch einige bisher unveröffentlichte Briefe und Bildmaterialien von Eco enthält.

Die ersten erwähnenswerten Dokumente führen uns in die späten 1960er Jahre, als die Internationale Vereinigung für semiotische Studien (IASSAIS) gegründet wurde. Eco gehörte zu den Gründungsmitgliedern und hat sich für die Organisation des ersten Weltkongresses für Semiotik eingesetzt. Als die Versuche, den Kongress in Osteuropa (genauer in Warschau) zu veranstalten, aus politischen Gründen scheiterten, bot er als Tagungsort Mailand an. Der erste Weltkongress fand hier 1974 statt (vgl. Chatman, Eco und Klinkenberg 1979) und bewirkte durch seine enthusiastische Atmosphäre, dass viele der internationalen Teilnehmer nach ihrer Rückkehr organisatorisch tätig wurden. So dauerte es nicht lange, bis mehrere nationale Semiotik-Gesellschaften ins Leben gerufen wurden. Bereits ein Jahr später fand an der Technischen Universität in Berlin der Gründungskongress der Deutschen Gesellschaft für Semiotik (DGS) statt, zu dem Eco als Plenarredner mit Überlegungen zu verschiedenen Arten der Zeichenbildung beitrug. Der Aufsatz wurde in dem Sammelband Zeichenprozesse. Semiotische Forschung in den Einzelwissenschaften (Posner und Reinecke 1977) veröffentlicht, dessen Erfolg die Veranstalter zur Gründung der vorliegenden Zeitschrift ermutigte.

Ecos Zusammenarbeit mit deutschen Semiotikern setzte sich bis zu seinem Tod fort. Im Semiotik-Archiv zeugen davon gemeinsame Konferenzbände und Projekte zur Semiotikgeschichte (z.B. Krampen u.a. 1981) sowie Programme von Tagungen, an denen Eco teilnahm, z.B. die deutsch-italienische Semiotik-Tagung im Stuttgarter Lindenmuseum (1986) oder das Colloquium mit und über Umberto Eco, das anlässlich seiner Ehrenpromotion an der Freien Universität Berlin 1998 stattfand. Im unsortierten ArchivTeil ruht die 1974 beginnende Korrespondenz zwischen Umberto Eco und Roland Posner, in der sich auch eine Zeichnung findet, welche auf die besagte Semiotik-Tagung in Stuttgart zurückgeht. ${ }^{2}$ Die Karikatur bewegt zu 
den in der Debatte um die Intentionalität von Zeichenprozessen bis dahin ungewöhnlichen Fragestellungen: Wie viel Zeit beansprucht die Rekonstruktion der Intentionen des Sprechers? Und was soll man eher anstreben - rekonstruieren oder reagieren? Eco reagierte mit dieser Karikatur auf den Beitrag von Roland Posner, und er reagierte verblüffend schnell, indem er die Karikatur noch während Posners Vortrags zeichnete, um sie dem Redner mit einem schelmischen Lächeln übergeben zu können. ${ }^{3}$

Posner plädierte im Vortrag für einen Kommunikationsbegriff, der das Vorliegen von Sender-Absichten auf mehreren Ebenen voraussetzt (vgl. Posner 1993). Für gelungene Kommunikation gilt dabei, dass der Empfänger nicht nur die Absicht des Senders erkennt, inn zu etwas zu bewegen (in diesem Fall zum Aufstehen), sondern auch erkennt, dass der Sender inm diese Absicht mitteilen will. Diesem Modell zufolge ist es keine Kommunikation, wenn etwa ein lautes Klatschen einer Lehrerin bewirkt, dass die Schüler unwillkürlich verstummen und zu ihr herschauen. Sie benutzt in diesem Fall das Klatschen als Signal, einen im Vergleich zur Kommunikation einfacheren Zeichentyp. Kommunikation im vollen Sinne liegt vor, wenn die Schüler leiser werden, weil sie dem Klatschen der Lehrerin ihren entsprechenden Wunsch entnommen haben. Die Verwendung von sprachlichen Zeichen ist meistens kommunikativ im engeren Sinne, etwa wenn die Lehrerin „Schaut her!" sagt und damit den Schülern die Möglichkeit gibt, ihre Absicht zu verstehen und sich dann zu entscheiden, ob sie sie befolgen (Posner 1993: 231-235).

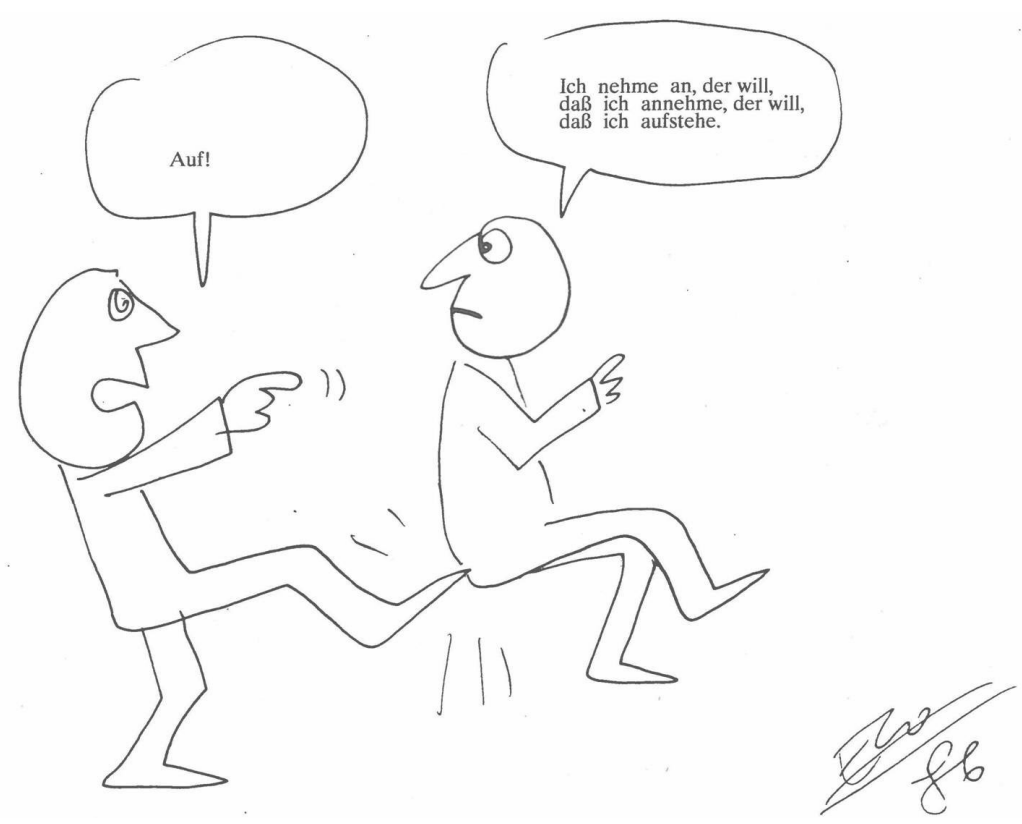

Abb. 1: Karikatur von Umberto Eco (1986). 
Eco parodiert in der Zeichnung die verschachtelten Formulierungen, die sich aus diesen Überlegungen ergeben. Dazu ist anzumerken, dass auch Eco sich über die Notwendigkeit der Annahme von Intentionalität in der Kommunikation ${ }^{4}$ durchaus im Klaren war, obwohl er sich nicht explizit auf die Tradition der intentionalistischen Semantik bezieht. Bereits in seinem Buch Zeichen. Einführung in einen Begriff und seine Geschichte (Eco 1973=1977) diskutiert er eine Reihe von möglichen Einteilungen der Zeichen, unter anderem nach den Intentionen und dem Bewusstseinsgrad des Senders. ${ }^{5}$ Die Komplexität der Zeichenprozesse demonstriert er anhand einer Reihe von geschickt gewählten Beispielsituationen mit einem Gastgeber, der einen Gast durch nervöses Trommeln mit den Fingern auf der Tischplatte loswerden möchte, was er einmal absichtlich tut, ein andermal unabsichtlich. Durch Beispiele mit einem Schauspieler, der den Gang eines Arthritikers nachahmt, gelangt Eco zu den Fällen, bei denen wir „nicht nur die Absicht berücksichtigen müßten, die der Empfänger dem Sender zuschreibt, sondern auch die Absicht, von der der Sender möchte, daß der Empfänger sie ihm zuschreibt“" (Eco 1973=1977: 49).

Vielleicht wollte Eco mit der Karikatur veranschaulichen, dass Kommunikation auch dann stattfindet, wenn wir uns zuerst nicht vollständig im Klaren sind, was der Sender uns kommunizieren wollte oder welche seine genauen kommunikativen Absichten sind. Das heißt: auch in alltäglichen Situationen verstehen wir prima facie ohne weitere Reflexion, was gesagt wird („Auf!"), und erst dann kommt die Rekonstruktion ex post. Hat er es ernst gemeint? Hab ich das richtig verstanden? Meinte er wirklich mich? Das implizite Verstehen ohne Bewusstwerdung der kommunikativen Intentionen stellt Eco humoristisch durch einen Tritt dar, dessen „Empfänger“ natürlich nicht so reflektiert reagieren wird, wie das intentionalistische Modell der Kommunikation es besagt. Allgemein gilt, dass Kommunikation nicht immer als eindeutige Kette von mentalen Zuschreibungen rekonstruiert werden kann, sondern oft mehrdeutig und offen in ihrer Interpretation ist.

Ecos Ironie hat im Übrigen selbst immer den Charakter einer offenen Kommunikation, in der eine klare Deutung nicht so leicht möglich ist. Man lacht über Ecos Scherz, bleibt aber doch mit einigen Fragen zurück: Hält Eco hier das Gegenteil des Gezeigten für wahr? Ist das eine Kritik oder inszeniert er nur, wie eine Kritik aussehen könnte? Sollen wir uns Kommunikation wirklich wie einen Tritt vorstellen, auf den man unwillkürlich reagiert? Oder vermischt er hier zwei unterschiedliche Zeichentypen - den Tritt als Signal und die reflektierte Kommunikation -, um uns zum Lachen zu bringen? Die interpretative Unsicherheit und Mehrdeutigkeit hatte oft in Ecos Scherzhaftigkeit eine elektrisierende Wirkung auf seine Zuhörer, weil dieser Humor immer eine wichtige Funktion erfüllte: Nicht nur schaffte er damit eine gesellige und offene Atmosphäre unter den Anwesenden, sondern er sah auch das Spielerische als Voraussetzung einer kritischen und antidogmatischen Haltung. In diesem Sinne kann auch sein Spott über eine Wissenschaftskultur, die es mit der Reflektiertheit schon mal übertreibt, verstanden werden. 
Auch Überlegungen über die Prinzipien des Komischen und die Einstellungen zum Lachen in unterschiedlichen Kulturen haben Eco lebenslang beschäftigt. Er befasste sich mit der Rolle des Lachens in literarischen Werken, etwa im Roman von Edmondo De Amicis // Cuore (Eco 1962), und kommentierte in Essays und Rezensionen traditionelle Komiktheorien wie z.B. von Luigi Pirandello oder Michail Bachtin (Eco 1980a und 1985). Einem klassischen Ansatz zur Komödie, dem zweiten Buch der Poetik von Aristoteles, ist übrigens die Handlung seines Romans Der Name der Rose verpflichtet. Die in Wirklichkeit verschollene Abhandlung wird in Ecos Roman im Bibliothekslabyrinth einer Benediktinerabtei versteckt - jedoch nicht so gut, dass die Neugierigen von ihrer (im Wortsinne) vergiftenden Wirkung verschont bleiben. Die Schrift wird am Ende zerstört, weil das Komische und das Lachen von denjenigen als gefährlich betrachtet werden, die eine dogmatische und geschlossene Weltsicht vertreten. Im Roman finden sich dabei Anspielungen auf Bachtins berühmte Schrift Rabelais und seine Welt. Volkskultur als Gegenkultur (1965=1987) sowie auf das Werk Rabelais', etwa durch die Erwähnung eines Textes von „Magister Alcofribas“, was auf Rabelais' Pseudonym Alcofribas Nasier hinweist (Eco 1980b=1982: 559; zu Bachtins und Rabelais' Einfluss auf Eco vgl. Stauder 2012: 23-32).

Es waren vor allem Bachtin und Pirandello, die Ecos eigene Überlegungen zur Komik und Humor beeinflusst haben - so hat Eco etwa im Sinne Pirandellos zwischen Komik (als Wahrnehmung des Gegensatzes) und Humor (als Fühlen des Gegensatzes, vgl. Eco 1981a) unterschieden. Als Ecos erster eigenständiger Beitrag zur Struktur komischer Texte kann der Vortrag Analisi di una "histoire drôle" auf dem von Carlo Bo und Roland Barthes organisierten Symposium in Urbino im Juli 1967 gelten (vgl. Todorov 1968). Viel stärker wurde allerdings von der semiotischen und linguistischen Humorforschung der kurze Essay II comico e la regola (Eco 1981a) rezipiert, der auf ein Kolloquium zur Rhetorik des Komischen in Brixen im Juli 1980 zurückgeht. ${ }^{6}$ Eco spricht von einem Regelbruch als Basis der Komik, wobei er den Begriff der Regel sehr weit fasst. Von der Tragik, die ebenfalls als Regelbruch gedeutet werden kann, unterscheide sich die Komik nicht primär durch ihre Themen, sondern vor allem durch die Tatsache, dass man sich bei der Art der gegebenen Regel weniger aufhält. Während eine Tragödie der Erklärung und Beschreibung der Regel in einem gegebenen sozialen Kontext großen Raum widmet, wird in den komischen Formen die Regel oft nicht einmal expliziert, weil sie für allgemein bekannt gehalten wird. Als Beispiel nennt Eco die Situation, in der sich Personen gegenseitig Torten ins Gesicht werfen: Dies wirkt komisch, weil man erwartet, dass Torten gegessen und nicht ins Gesicht geworfen werden. Weiter führt Eco die Verletzung der Grice'schen Konversationsmaximen (Grice 1975) als häufige Basis der Komik in dialogisch konzipierten Komikformen an, insbesondere in Witzen. Die Rolle des Regelbruchs behandelt Eco ebenfalls im Aufsatz The frames of comic 'freedom'(1984a), der dem Karneval gewidmet ist und Bachtins Ideen weiterführt. Eco sieht den Karneval als autorisierte Transgression an, was jedoch, wie er im selben Atemzug 
hinzufügt, „a blatant case of contradictio in adjecto“ ist (Eco 1984a: 6; Kursivierung im Original).

Der Streifzug durch das Semiotik-Archiv führt uns schließlich zum Regal mit Handbüchern. Wie seine Abhandlungen verraten, war Eco fasziniert von Listen und Lexika aller Art, daher überrascht es nicht, dass er sich aktiv an der Gestaltung von semiotischen Handbüchern und Lexika beteiligte. Er war Mitglied des wissenschaftlichen Beirats des Encyclopedic Dictionary of Semiotics (Sebeok 1986), für das er mehrere Einträge schrieb. Für das vierbändige Handbuch Semiotik. Ein Handbuch zu den zeichentheoretischen Grundlagen von Natur und Kultur (Posner, Robering und Sebeok 1997-2004) lieferte er je einen Beitrag über die Geschichtsschreibung der Semiotik (Eco 1997) und über Fälschungen in Kunst und Kunstgewerbe (Eco 2004). Er war der einzige unter den fast zweihundert Beiträgern des Handbuchs, dessen Werk zugleich auch ein eigener Artikel gewidmet ist (Proni 1998). Diese Praxis wurde seitdem fortgesetzt - so finden wir beispielsweise in der 3. Auflage des Encyclopaedic Dictionary of Semiotics (Sebeok und Danesi 2010) neben Einträgen von Eco auch einen Eintrag über Eco.

Nun stellt sich die Frage, wann die Eco-Institutionalisierung im semiotischen Bereich einsetzte und wie dieser Prozess vonstattenging. Der Anfang von Ecos internationaler Karriere fällt ins Ende der 1960 Jahre: Nach 1969 besuchte er als „reisender Semiotiker“ (Proni 1988: 15) mehrere nord- und südamerikanische Universitäten, und mit Hilfe dieser Kontakte konnte er den ersten Weltkongress der IASS-AIS in Mailand organisieren. Die 1970er Jahre brachten bereits eine Reihe von Übersetzungen seiner zeichentheoretischen Werke, insbesondere ins Deutsche und ins Englische. ${ }^{7}$ Die Arbeit an Autorisierungen von Übersetzungen seines Buchs La struttura assente motivierte inn zu weiteren zeichentheoretischen Abhandlungen (Eco 1971 und 1973) und führte schließlich zur $A$ Theory of Semiotics, die inm internationale Bekanntheit einbrachte (Eco 1976). Zu diesem Zeitpunkt erhielt Eco, der 15 Jahre zuvor zum Privatdozenten für Ästhetik an der Universität Turin ernannt wurde, eine ordentliche Professur für Semiotik an der Universität Bologna. ${ }^{8}$ Nach dem Erscheinen des Romans Der Name der Rose (1980b), der als kreatives Ergebnis angewandter Semiotik angesehen werden kann (Nöth 2000: 126), galt Eco weltweit als Personifizierung der Semiotik. Als er 1981 für das Buch Die Welt als Zeichen. Klassiker der modernen Semiotik (Krampen u.a. 1981) einen Text über Roman Jakobson $^{9}$ schrieb, gehörte er bereits selbst zu den Klassikern.

$\mathrm{Da}$ Eco nie ein ,bloßer Akteur' war, sondern stets auch ein Beobachter, äußerte er sich in seinen Texten und Interviews auch zu den Tücken dieses Institutionalisierungsprozesses. Aus den Kolumnen für das italienische Wochenmagazin Espresso, aus denen mehrere humorvolle Textsammlungen hervorgingen (vgl. Eco 2001), erfahren wir viel über den Altag eines öffentlichen Intellektuellen, angefangen beim Interviewzwang bis hin zur Beschäftigung mit neuen Büchern, die inm, der längst als Autorität galt, täglich zugesandt wurden. 
Für die weltweite semiotische Forschung hinterlässt Eco in mehrfacher Hinsicht eine Lücke, die nicht gefüllt werden kann. In Zukunft wird das Werk Ecos für den Wissenschaftler und Schriftsteller stehen müssen: Immerhin erlaubt es Ecos Konzept des „opera aperta“, des offenen (Kunst-)Werks, mit seinen Schriften dauerhaft in einen Dialog zu treten und innen immer neue Bedeutungen zu entlocken. Ecos Arbeiten stellen dabei einen eigenständigen Strang innerhalb der semiotischen Forschung dar, auf den auch heute noch in zahlreichen Publikationen Bezug genommen wird, die an seine Theorien anknüpfen, sie weiter entwickeln und auf neue Fragestellungen anwenden. Schließlich reichen die mit seinem Namen verbundenen Assoziationen von der Semiotik, der Ästhetik und der Mediävistik über die postmoderne Literaturtheorie und eben die Komik- und Comicforschung bis hin zur Medientheorie und zur theoretischen Reflexion des Faschismus. $\mathrm{Zu}$ allen diesen Bereichen hat er eigenständige Konzeptionen und tiefgehende Untersuchungen beigetragen, die bis heute nichts von ihrem anregenden Potential verloren haben.

Zugleich erscheint Ecos Rang als einer der großen Romanciers des späten 20. Jahrhunderts gesichert - bereits Der Name der Rose und Das Foucaultsche Pendel hätten hierzu ausgereicht, doch Eco ließ innen noch fünf weitere Romane folgen. Nun ist Eco nicht der einzige Wissenschaftler, der auch erfolgreich literarisch tätig war. Als Besonderheit kann aber gelten, dass er nicht nur in zwei verschiedenen Gebieten Herausragendes leistete, sondern dass sein literarisches Werk mit seinem wissenschaftlichen untrennbar verbunden ist: Weder das eine noch das andere kann für sich allein vollständig entschlüsselt werden, sind doch die literarischen Kunstwerke und die wissenschaftlichen Schriften Ecos in ihren Konzeptionen und Reflexionen verflochten und verweisen in ihren Gedankenwelten aufeinander. Wissenschaft und Kunst erscheinen im Werk von Umberto Eco nicht als zwei getrennte Sphären, für die man - wie es zunehmend üblich ist - getrennte Hochschulen, Bibliotheken und Förderprogramme braucht. Dies bindet inn in den zeitgenössischen Kontext der Postmoderne ein, in der sein Werk jedoch keineswegs aufgeht, und verweist zugleich zurück auf die von inm so geschätzte Gedankenwelt des Mittelalters. Ebenso wie im Mittelalter Literatur und Kunst, Erfindung und Erforschung, Spiel und Experiment noch nicht zu trennen sind, macht dies auch bei Ecos Werk keinen Sinn.

Aliquando praeterea rideo, iocor, ludo, homo sum.

So heißt es in Ecos berühmtestem Buch, und obwohl dieses Zitat dort selbst schon ein Zitat und das Kunstwerk interpretativ offen ist, liegt es doch nahe, dass wir mit der Aussage von Plinius dem Jüngeren sympathisieren dürfen, der sich erst bei Lachen und Spiel als Mensch empfand. Für Eco hatten Wissenschaft und Literatur gleichermaßen den Charakter eines Spiels, bei dem herausfordernd gelacht oder leise geschmunzelt werden darf, gerade wenn es intellektuell anspruchsvoll zugeht - auch dies ist sicher eine 
Botschaft der oben abgebildeten Karikatur. Vermutlich hätte es Eco gefallen, dass einer seiner zahllosen Nachrufe in einem Heft über Lachen als Zeichenprozess erscheint.

\section{Anmerkungen}

12005 wurde Eco in einer vielzitierten Liste der wichtigsten öffentlichen Intellektuellen, die von den Magazinen Prospect und Foreign Policy gemeinsam publiziert wurde, auf den zweiten Platz gewählt, gleich hinter Noam Chomsky und noch vor Richard Dawkins, Václav Havel, Jürgen Habermas und Salman Rushdie. Die Leser der beiden Magazine konnten dabei aus einer Liste von 100 Intellektuellen wählen, die von den Redaktionen zusammengestellt worden war.

2 Zu Ecos Karikaturen aus den 1950er Jahren vgl. Stauder 2004.

3 Den Hinweis auf die Karikatur und Informationen zu ihrer Entstehung verdanken wir Roland Posner. Ecos Text ist in Posners deutscher Übersetzung überklebt worden.

4 Zur Rolle der Zuschreibung von intentionalen Zuständen sowohl in der semiotischen Forschung als auch in der gegenwärtigen neuro- und entwicklungspsychologischen Forschung siehe Arielli 2012.

5 Diese Überlegungen finden wir in gekürzter Form auch im einleitenden Kapitel von A Theory of Semiotics (Eco 1976).

6 Zu diesem Essay und seiner Rezeption siehe Attardo (1994: 177ff.), zu Ecos Regelkonzept vgl. Wirth (1999: 26).

7 So konnte man in den 1970er und 1980er Jahren feststellen, dass die grundlegenden Werke seiner Semiotik ungewöhnlich schnell ins Deutsche übersetzt wurden (vgl. Eschbach und Eschbach-Szabó 1986: 198ff.), wofür sich insbesondere Günter Memmert und Jürgen Trabant einsetzten

8 Zu den Forschungsaktivitäten der Bologneser Semiotiker Ende der 1970er und Anfang der 1980er Jahre siehe den Bericht von Proni (1987). Eco war an der Gestaltung von modernen Studiengängen der Kommunikationswissenschaft beteiligt. Der erste Studiengang dieser Art wurde 1993 in Bologna unter seiner Leitung eingerichtet.

9 Jakobson hielt auf dem von Eco organisierten Mailänder Weltkongress die Eröffnungsrede (siehe Jakobson 1979), die Eco 1981 als den einzigen bibliographischen Eintrag von Jakobson einstuft, der deutlich als semiotisch erkennbar sei (Eco 1981b: 178).

\section{Literatur}

Arielli, Emanuele (2012), „Thinking about complex mental states: language, symbolic activity and theories of mind“". In: Hess-Lüttich, Ernest W. B. (Hrsg.), Sign Culture - Zeichen Kultur. Würzburg: Königshausen \& Neumann: 491-501.

Attardo, Salvatore (1994), Linguistic Theories of Humor. Berlin und New York: De Gruyter. Bachtin, Michail M. (1965), Rabelais und seine Welt. Volkskultur als Gegenkultur. Aus dem Russischen übersetzt von G. Leupold. Frankfurt am Main: Suhrkamp 1987. Chatman, Seymour, Umberto Eco und Jean-Marie Klinkenberg (Hrsg.) (1979), A semi- 
otic landscape: proceedings of the First Congress of the International Association for Semiotic Studies, Milan, June 1974. Berlin und New York: De Gruyter.

Eco, Umberto (1962), „Franti o il Cuore“. Il Caffè 3. Abgedruckt als „Elogio di Franti“ in: Umberto Eco, Diario minimo. Milano: Mondadori 1988: 85-96.

Eco, Umberto (1968), La struttura assente: La ricerca semiotica e il metodo strutturale. Milano: Bompiani. Deutsch von J. Trabant: Einführung in die Semiotik. München: Fink 1972.

Eco, Umberto (1971), Le forme del contenuto. Milano: Bompiani.

Eco, Umberto (1973), II segno. Milano: Istituto Editoriale Internazionale. Deutsch von G. Memmert: Zeichen. Einführung in einen Begriff und seine Geschichte. Frankfurt am Main: Suhrkamp 1977.

Eco, Umberto (1976), A Theory of Semiotics. Bloomington und London: Indiana University Press. Deutsch von G. Memmert: Semiotik. Entwurf einer Theorie der Zeichen. München: Fink 1987.

Eco, Umberto (1977), „Arten der Zeichenbildung“. In: Posner und Reinecke 1977: 58-68.

Eco, Umberto (1980a), „Corpo Dio“. Espresso 3.2.1980: 68-72.

Eco, Umberto (1980b), I/ nome della rosa. Milano: Bompiani. Deutsch von B. Kroeber: Der Name der Rose. München u.a.: Hanser 1982.

Eco, Umberto (1981a), „Il comico e la regola“. Alfabeta, 21.02.1981, nachgedruckt in: Umberto Eco, Sette anni di desiderio. Milano: Bompiani 1993: 253-260.

Eco, Umberto (1981b), „Der Einfluss Roman Jakobsons auf moderne Semiotik“. In: Krampen u.a. 1981: 173-204.

Eco, Umberto (1984a), Semiotics and the Philosophy of Language. Bloomington, IN: Indiana University Press.

Eco, Umberto (1984b), „The frames of comic 'freedom'“. In: Umberto Eco, Viacheslav V. Ivanov und Monika Rector (Hrsg.), Carnival! Berlin und New York: De Gruyter: 1-9.

Eco, Umberto (1985), „Pirandello ridens“. In: ders.: Sugli specchi e altri saggi. Milano: Bompiani: 261-269.

Eco, Umberto (1997), „History and historiography of semiotics“. In: Posner u.a. 19972004, Bd. 1: 700-746.

Eco, Umberto (2001), Sämtliche Glossen und Parodien. München: Hanser.

Eco, Umberto (2004), „Fakes in arts and crafts“. In: Posner u.a. 1997-2004, Bd. 4: 35713580.

Eschbach, Achim und Viktoria Eschbach-Szabó (1986), Bibliography of Semiotics 19751985. Bd 1. Amsterdam (Philadelphia): John Benjamins.

Grice, Herbert P. (1975), „Logic and Conversation“. In: Peter Cole und Jerry L. Morgan (Hrsg.), Syntax and Semantics. New York: Academic: 41-58.

Jakobson, Roman (1979), „Coup d'oeil sur le développement de la sémiotique“. In: Chatman u.a. 1979: 3-19.

Krampen, Martin, Klaus Oehler, Roland Posner und Thure von Uexküll (Hrsg.) (1981), Die Welt als Zeichen. Klassiker der modernen Semiotik. Berlin: Severin und Siedler.

Nöth, Winfried (2000), Handbuch der Semiotik. 2. Auflage. Stuttgart und Weimar: Metzler. Posner, Roland (1993), „Believing, Causing, Intending: The Basis for a Hierarchy of Sign Concepts in the Reconstruction of Communication". In: René J. Jorna, Barend van Heusden und Roland Posner (Hrsg.), Signs, Search and Communication: Semiotic Aspects of Artificial Intelligence. Berlin und New York: De Gruyter: 215-270. 
Posner, Roland und Hans P. Reinecke (Hrsg.) (1977), Zeichenprozesse. Semiotische Forschung in den Einzelwissenschaften. Wiesbaden: Athenaion.

Posner, Roland, Klaus Robering und Thomas A. Sebeok (Hrsg.) (1997-2004), Semiotik. Ein Handbuch zu den zeichentheoretischen Grundlagen von Natur und Kultur. 4 Bde. Berlin und New York: De Gruyter.

Proni, Giampaolo (1987), „Semiotic Studies in Bologna: A Report“. In: Thomas A. Sebeok und Jean Umiker-Sebeok (Hrsg.), The Semiotic Web 1986. Berlin und New York: De Gruyter: 603-617.

Proni, Giampaolo (1988), „Umberto Eco: An Intellectual Biography“. In: Thomas A. Sebeok und Jean Umiker-Sebeok (Hrsg.), The Semiotic Web 1987. Berlin und New York: De Gruyter.

Proni, Giampaolo (1998), „The position of Eco“. In: Posner u.a. 1997-2004, Bd. 2: 23112320.

Sebeok, Thomas A. (Hrsg.) (1986), Encyclopedic Dictionary of Semiotics. 3 Bde. Berlin und New York: De Gruyter.

Sebeok, Thomas A. und Marcel Danesi (Hrsg.) (2010), Encyclopedic Dictionary of Semiotics. 3. Auflage. 3 Bde. Berlin und New York: De Gruyter.

Stauder, Thomas (2004), „Umberto Eco als Karikaturist (Filosofi in libertà, 1958)“. Zibaldone. Zeitschrift für italienische Kultur der Gegenwart 38: 97-113.

Stauder, Thomas (2012), Gespräche mit Eco aus drei Jahrzehnten. Berlin: LIT.

Todorov, Tzvetan (1968), „L'analyse du récit à Urbino“. Communications 11, 1: 165-167.

Wirth, Uwe (1999), Diskursive Dummheit. Abduktion und Komik als Grenzphänomene des Verstehens. Heidelberg: Winter.

Dr. Martin Siefkes

Technische Universität Chemnitz

Philosophische Fakultät

Institut für Germanistik und Kommunikation

Straße der Nationen 62

D-09111 Chemnitz

E-Mail: martin.siefkes@phil.tu-chemnitz.de
Mgr. Veronika Opletalová, Ph.D. Univerzita Palackého v Olomouci Filozofická fakulta

Katedra germanistiky

Křížkovského 10

CZ-77147 Olomouc

E-Mail: veronika.opletalova@upol.cz

Prof. Dr. Emanuele Arielli

Università IUAV di Venezia

Dipartimento di Culture del progetto

Cotonificio veneziano

Dorsoduro 2196

I-30123 Venezia

E-Mail: emanuele.arielli@gmail.com 